\title{
A reflection on the Mohr failure criterion
}

\author{
Shuguang Li \\ Faculty of Engineering, University of Nottingham \\ Nottingham NG8 1BB, UK
}

\begin{abstract}
The Mohr failure criterion has been re-examined and re-presented in a rational manner in the context of brittle materials. The generic assumptions and their logical inferences have been elaborated, as well as the basic assumption of the isotropy of the material. It has been revealed for the first time that the assumption that 'failure is determined by the stresses exposed on the fracture plane' relies on the basic assumption of isotropy and its adoption in the establishment of popular modern failure criteria for anisotropic composites is therefore deemed to be out of context. Based on the generic assumptions and well-established experimental observations on the fracture of brittle materials under uniaxial tension, pure shear and uniaxial compression and through rational reasoning, the characteristics of the failure envelope on the tensile side have been deduced, revealing many features which have never been fully established previously. From the perspective of the Mohr criterion, the Coulomb-Mohr criterion has been given a thorough and objective appraisal, leading to a complete dismissal of it as a criterion of practical relevance.
\end{abstract}

Keywords: Failure criterion; The Mohr Criterion; Mohr's circle; The Coulomb-Mohr criterion; Brittle materials; Failure envelope; Fracture plane.

\section{$1 \quad$ Introduction}

Material failure has been a critical issue in applications ever since materials was employed in structures to sustain loads. One of the earliest attempts of addressing the issue goes centuries back by Coulomb [1] when he proposed a friction-type model taking due consideration of the shear and direct stresses on the fracture plane. Coulomb's model was rejuvenated into the Coulomb-Mohr (C-M) criterion [2], or Mohr-Coulomb, as it is commonly known nowadays 
after the Mohr criterion was published at the turn of the previous century [3]. Due to its simplicity, C-M has attracted a considerable amount of attention and applications accordingly. However, a comprehensive appraisal of it is not readily available to the best of the author's knowledge, whilst in [2] an anomaly of its allowance for infinite distortion without causing failure was revealed leading to a dismissive conclusion on validity of the criterion.

The original Mohr criterion did not have to suffer from the anomaly as C-M did. It relied on an experimentally obtained failure envelope for a given material which circumscribes the major Mohr's circles at failure under all possible stress states. The envelope was usually curved in general. In his original paper [3], Mohr did swiftly move to the straight line simplification and consequently it lined up with Coulomb's criterion almost by default, though not by Mohr himself [3].

Relevant appraisals of the Mohr criterion (not C-M) are even scarcer in the literature, although mentioning it in textbooks has been a common practice, e.g. [4], where the account has usually been kept very brief, if any, before slipping to the $\mathrm{C}-\mathrm{M}$ for elaborations. On the other hand, a key assumption introduced in the Mohr criterion, viz. 'failure is determined by the stresses exposed on the fracture plane', has been adopted in many modern developments, some are rather influential, in particular, for modern composites, e.g. [5,6,7], which will be revealed in this paper as a leap of faith without appropriate justifications. An appropriate reflection on the Mohr criterion is therefore desirable. As Mohr's original paper was published over a hundred years ago and it was in German (The author did search for an English version of it but to no vail), it will become more and more unlikely for researchers nowadays to retreat back to it. Through the present paper, the author intends to re-present the Mohr criterion in a rational manner before fully exploring the logical implications of the assumptions introduced in the criterion. Consequently, the presentation here is not meant to be identical to what was presented in [3] originally.

After achieving the above, the present paper aims to clarify the following issues which are a bit loose in the literature one way or another.

(1) The relationship between the failure envelope and the fracture plane;

(2) A rational assessment on the applicability of the assumption that failure is determined by the stresses exposed on the fracture plane;

(3) The characteristics on the tensile side of the failure envelope; and

(4) An appropriate appraisal of C-M. 
A comprehensive literature survey is not to be pursued in this paper, whilst detailed historical account can be found in [8] on the subject of strength theories, because most publications on relevant topic tended to be biased to the C-M, e.g. [2,4,8-11], and the majority of them were about applications of C-M in specific problems. They are of little relevance to the subject of the present paper. However, the Extended Mohr-Coulomb (EMC) model as presented in [12] is of help to some of the elaborations in this paper. In this model, the questionable linear relationship between the shear and direct stresses as was in the conventional C-M had been replaced by an exponential function. It seemed to fit well to the experimental data on the compressive side where the model was introduced for primarily. More detailed discussion will be provided later in this paper.

\section{The generic assumptions in the Mohr criterion}

\subsection{Mohr's circles}

The Mohr criterion rests heavily on the concept of Mohr's circles, especially their generalisation for 3D stress states involving a set of three circles completely defined by the stress state at a material point concerned using its principal stresses $\sigma_{1}, \sigma_{2}$ and $\sigma_{3}$ (in descending order). For the convenience of subsequent references, they are briefly introduced here, although the details can be found in textbooks, as illustrated in Fig. 1. The outmost circle is called the major Mohr's circle determined by $\sigma_{1}$ and $\sigma_{3}$. Any point $\mathrm{P}$ on the $\sigma-\tau$ plane within the shaded zone carries two pieces of information: (i) the orientation of a plane whose direction cosines are directly related to the distances from $\mathrm{P}$ to the centres of the three Mohr's circles involved, and (ii) the coordinates $(\sigma, \tau)$ of $\mathrm{P}$ in the $\sigma-\tau$ plane correspond to the direct and shear stresses on that plane. Points outside the shaded part are not associated with the given stress state.

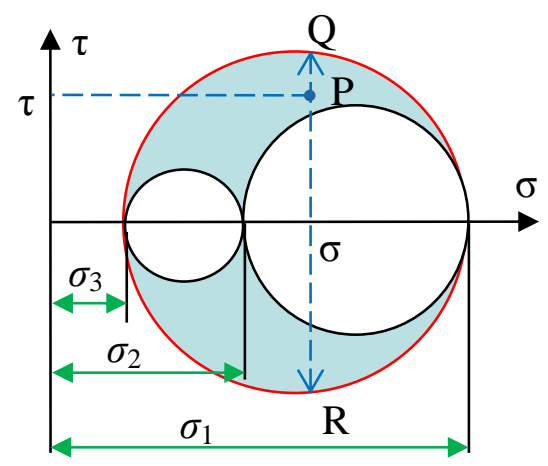

Fig. 1 Mohr's circles for a 3D stress state 


\subsection{Generic assumptions of the Mohr criterion}

In the Mohr criterion, conventional assumptions as in solid mechanics, in particular, the isotropy of the materials concerned, are adopted, in addition to the brittleness of the material, i.e. the material exhibits different strength against tension and compression. They will be referred to as the basic assumptions. Re-presenting the Mohr criterion to the best of the author's understanding and rationalisation of Mohr's original paper [3], it can be reconstructed based on the following generic assumptions specifically introduced for this criterion.

1. Failure/fracture takes place on a plane through the material point concerned.

2. Failure is determined by the stresses exposed on the fracture plane.

3. Shear stress on any plane always tends to promote fracture on this plane.

4. There exists an envelope which circumscribes the major Mohr's circles for all stress states at failure.

5. The envelope obtained above offers a criterion for the fracture of a plane as a point on the major Mohr's circle of a stress state, i.e. any point outside the envelope is associated with a failed plane whilst the plane corresponding to a point inside the envelope remains safe.

The Mohr criterion should only be applied in the context of the assumptions as provided above. These assumptions can be elaborated individually as follows whilst their more profound logical inferences will be presented in the next subsection.

Assumption 1 is certainly applicable to brittle materials where failure is usually in a form of fracture on a plane but it should not be taken for granted in general for other materials. Ductile materials tend to yield, which may not always be confined within a plane. The Mohr criterion is therefore applicable primarily to brittle materials.

Assumption 2 narrows the scope from a stress state involving six independent components to the direct and shear stresses on the potential fracture plane in order to assess the possible failure of the material, although the magnitudes of these stresses and the orientation of the plane are yet to be determined. This is justified through Mohr's circles as follows. Any plane corresponds to a point as shown in Fig. 1 and the point is associated with a direct and a shear stress. A material fails if any plane fails and it is safe if none of the planes fails. What this assumption leads to is the search for the most critical plane under the given stress state. 
For a given stress state as graphically represented by the Mohr's circles in Fig. 1, assumption 3 states that, the higher the value of shear stress, the more prone to failure and therefore amongst all planes sharing the common direct stress, e.g. that of point P, i.e. amongst all points along the vertical line QPR within the shaded part, planes bearing the maximum magnitude of shear stress are the most critical, i.e. Q and R on the major Mohr's circle. Therefore, the potential fracture planes are always associated with points on the major Mohr's circle.

The existence of a failure envelope as illustrated by M-M and N-N in Fig. 2, symmetrically placed on either side of the $\sigma$ axis, as introduced in assumption 4 deserves some elaboration. There are two aspects involved, practical and mathematical (so described for argument's sake). The practical existence refers to a hypothetic premise that, when the major Mohr's circles for all possible stress states at failure were collected on the $\sigma$ - $\tau$ plane, a unique envelope could be constructed that should circumscribe every single Mohr's circle involved. The objective of the present paper is not to come up with a new criterion but to re-present the Mohr criterion so that it will be rational, at least, free from obvious inconsistencies. Therefore, the practical existence will be inherited, i.e. no major Mohr's circle under any stress state at failure would be found not in touch with the envelope. The other aspect is its mathematical existence. Improper formulation could easily deny the existence of a solution to a mathematical problem. In the present case, the practical existence of the envelope has determined the tangent to the envelope already. As an envelope mathematically, the tangential point and the tangent itself are determined collectively by the Mohr's circle concerned and its neighbouring ones. In Mohr's original paper [3], it had not been made crystal clear that the tangent to the envelope was associated with the orientation of the fracture plane, but it was certainly strongly implied at least. Imagine that one was to construct the envelope experimentally. From each test, one would have two pieces of information, a major Mohr's circle as the measure of the strength under the stress state and the fracture plane orientation which would specify the tangential point of the envelope to the Mohr's circle. This is mathematically conflicting as the tangent would be determined twice by two different means. Any mismatch there would dismiss the mathematical existence of such an envelope. This mathematical aspect of the existence of the envelope is a part of the rationalisation to be made in this paper. It is a pure matter of mathematical formulation of the problem and hence a matter of presentation of various assumptions. In other word, the practical existence of the envelope and the orientation of the fracture plane cannot be 
assumed at the same time, and the latter should be a by-product of the former. Generic assumption 4 has therefore been present without mentioning the fracture plane.

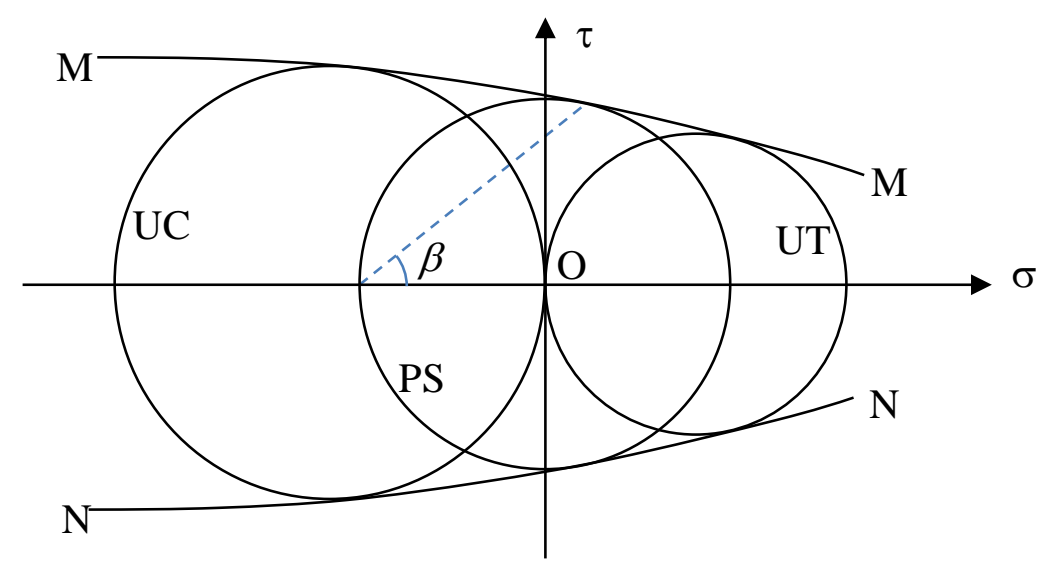

Fig. 2 The major Mohr's circles at failure under typical stress states and the failure envelope

As a mathematical artefact, the envelope alone does not come with definitive and necessary physical meaning, apart from being an envelope. Its relationship to the fracture plane has been left somewhat loose, a point most accounts on the Mohr criterion in the literature tended either to shy away from it, e.g. [4], or to take for granted that it identifies the orientation of the fracture plane, e.g. [11].

To clarify the position, it is essential to distinguish the fracture of a plane as the subject of assumption 5 and the failure of a material point under a stress state (represented by the major Mohr's circle). If a plane is safe, it does not guarantee the survival of the material under the stress state because failure might take place on other planes passing the same material point. A natural question to ask would be which plane is most critical. Given assumption 2, the actual determination of the fracture of a plane requires a criterion to assess what combination of the two stresses exposed on a plane would trigger the fracture of the plane. Assumption 5 offers exactly this. The original Mohr's criterion [3] had not provided a straight answer but implied this. It will be made explicit in this paper, as will be seen later that this helps to avoid the conflict as stated above. The envelope as was obtained from material failure based on the stress state as represented by its major Mohr's circle has been employed as a criterion for the fracture of a plane based on the direct and shear stresses on the plane. Any conflict as described above can be attributed to either experimental error or inapplicability of one or more assumptions to the material concerned. 
The generic assumptions as presented above are apparently mutually independent.

\subsection{Logical inferences of the generic assumptions}

The Mohr criterion has often been presented in the literature involving assumptions expressed differently from the generic assumptions as presented in the previous subsection. Some of them will be proven here to be equivalent expressions as the logical inferences of the generic assumptions. From assumptions 1 to 3 , one can deduce the following two equivalent inferences:

I. The pair of planes, such as those represented by points $\mathrm{Q}$ and $\mathrm{R}$ on the major Mohr's circle, as shown in Fig. 1, are equally critical. They are both parallel to the direction of the intermediate principal stress $\sigma_{2}$ and they are at an equal angle to the $1^{\text {st }}$ principal directions, as well as the $3^{\text {rd }}$.

This is so because at a given stress state and a given direct stress, the magnitude of the shear stress can only be as high as that of Q and R on the major Mohr's circle, with $\mathrm{Q}$ and $\mathrm{R}$ being symmetric about the $\sigma$-axis. If failure takes place at this value of direct stress, it will have to be equally likely on the planes as represented by $\mathrm{Q}$ and $\mathrm{R}$, symmetric about the directions of the $1^{\text {st }}$ and the $3^{\text {rd }}$ principal stresses.

II. The intermediate principal stress does not contribute to the failure.

Different values of the intermediate principal stress will not make any difference to the major Mohr's circle. If fracture is associated only with the major Mohr's circle, whatever value the intermediate principal stress takes will not make any difference.

Inferences I and II are both implied by assumptions 1 to 3. Logically, I or II is equivalent to assumptions 1 to 3 , stating the same premise that failure takes place on a plane and is dictated by the major Mohr's circle.

The above inferences are often seen in the literature, sometimes with a little justification and sometimes not. There is another important inference about the fracture plane. Mohr stated it in his original paper [3] in a vague manner and out of his intuition rather than logic. A logical deduction of the orientation of the fracture surface from the generic assumptions is therefore presented as follows to conclude this aspect of the Mohr criterion. 
If the fracture of a plane is determined entirely by the direct and shear stresses on this plane, a stress state is safe only if its major Mohr's circle is completely contained within the envelope, e.g. $S_{1}$ in Fig. 3, as none of its plane will go beyond the failure envelope according to assumption 5. Any stress state whose major Mohr's circle goes beyond the envelope is deemed to have failed, e.g. $\mathrm{S}_{2}$ in Fig. 3 as some of the planes have failed. When the major Mohr's circle becomes tangential to the envelope, e.g. $S$ in Fig. 3, there are only two tangential points, e.g. $\mathrm{T}_{1}$ and $\mathrm{T}_{2}$ in Fig. 3, as mirror images each other about the $\sigma$ axis, representing two planes orientated at $\pm \theta$ from the direction of the $1^{\text {st }}$ principal stress, where the fracture criterion for the plane is met. They and only they will be critical to fracture and they must be the fracture planes under the stress state concerned. Therefore, one has the inference from generic assumption 5 as follows.

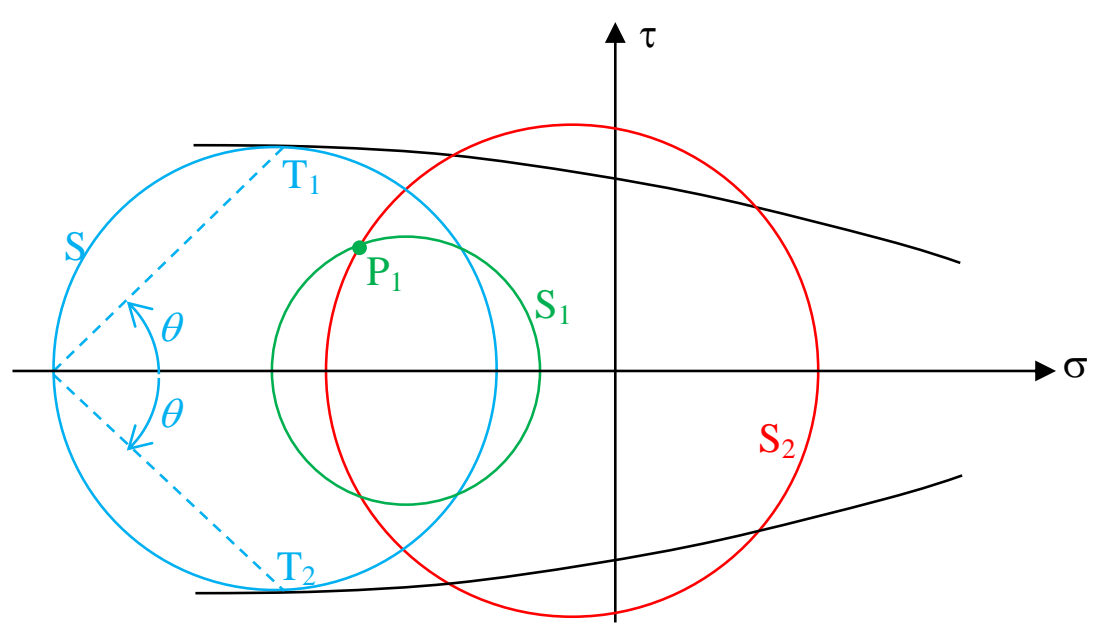

Fig. 3 Relationship between a surviving/failing stress state and a surviving/failing plane

III. The plane associated with the point on the major Mohr's circle tangential to the failure envelope defines the fracture plane for the stress state represented by the major Mohr's circle.

The above relationship between the fracture plane and the tangential point to the major Mohr's circle has been made use of in existing applications of the Mohr criterion whilst taking it for granted. There has been lack of a well-documented justification as above in the literature, to the best of the author's knowledge. The closest point of reference is the case of $\mathrm{C}-\mathrm{M}$ where the tangential point has been associated with the fracture plane. This is the case because it had been preceded by the Coulomb criterion [1] which was proposed based on the 
fracture plane as a starting point. On that plane the shear and direct stresses were related in a simple relationship as Coulomb proposed. In the Mohr criterion, without assumption 5, the envelope as introduced in assumption 4 is for the stress state, not related to the fracture plane directly. With inference III, supported by assumption 4, the fracture plane can be determined in the Mohr criterion in a similar way as in C-M, but the failure envelope does not have to be a straight line as in C-M.

With the assumptions of the Mohr criterion stated and their implications elaborated, the Mohr criterion can be presented as follows. Given the envelope for the material as described in assumption 4, any stress state of the material is safe if and only if its major Mohr's circle is inside the envelope. A stress state is critical when its major Mohr's circle inscribes the envelope whilst the circumferential angle in the major Mohr's circle between the tangential point and the $1^{\text {st }}$ principal stress identifies the orientation of the fracture plane relative to the direction of the $1^{\text {st }}$ principal stress.

\subsection{Reflection on the basic assumption of isotropy}

At this point, it is worth reflecting the basic assumption of isotropy of the material under consideration. This assumption allows the material to choose its critical plane to fracture according to the stress state, and the stress state only. Generic assumption 3 delivers the premise that the major Mohr's circle is representative only under the assumption of isotropy, as far as the failure is concerned within the Mohr criterion. In absence of isotropy, this process of self-identification of the critical plane will no longer apply as the material has relatively stronger and weaker directions due to its anisotropy which is completely independent of the stress state. In case of unidirectionally fibre reinforced composites, for instance, a plane perpendicular to fibres can usually take a much higher shear stress than a plane parallel to fibres in presence of direct tensile stresses of the same magnitude on these planes, respectively.

All stress components involved in a stress state should make contributions to the failure of the material under the stress state, in general. In the case where the failure can be determined by some of the stress components, e.g. when the maximum principal stress, the Tresca, or the Mohr criterion becomes applicable, these stress components have to be sufficiently representative. Their representativeness relies on the isotropy of the materials, i.e. the material gives the freedom to the stress state alone to decide the fracture plane. Generic assumption 2 in the Mohr criterion complemented by generic assumption 3 allows stresses to 
elect the most representative components to cause the fracture and consequently to appear on the fracture plane. This helps to neglect the contributions from other stress components making relatively insignificant contributions, which are encapsulated into the intermediate principal stress when the stress state is expressed in terms of principal stresses. If this selfselection process is interfered by other considerations, such as materials anisotropy, the components exposed on the fracture plane will no longer be as representative as they would otherwise, as far as failure is concerned. The contribution from other stress components will therefore no longer be negligible.

The self-selection process and its inapplicability to anisotropic materials can also be easily illustrated as follows, assuming principal stresses being $\sigma_{x}, \sigma_{y}$ and $\sigma_{z}\left(\sigma_{x} \geq \sigma_{y} \geq \sigma_{z}\right.$ for the time being). In an isotropic material, the fracture plane will be parallel to $\sigma_{y}$. In this case, the magnitude of $\sigma_{y}$ is allowed to vary without affecting the fracture plane provided that it only varies between $\sigma_{x}$ and $\sigma_{z}$. If $\sigma_{y}$ reduces below $\sigma_{z}$, the fracture plane will be readjusted to be parallel to $\sigma_{z}$ since $\sigma_{z}$ is now the intermediate principal stress. Similarly, if $\sigma_{y}$ increases above $\sigma_{x}$, the fracture plane will be parallel to $\sigma_{x}$ instead. The process as described is spontaneous and it takes place only if the material is isotropic when there is no other preference of potential fracture plane from the material itself. With the fracture plane prefixed, e.g. to be parallel to $\sigma_{x}$, due to the consideration of anisotropy, such self-adjustment will no longer take place, at least not based on the stresses only. It will be even more obvious if one is reminded that an anisotropic material does not always have principal axes and even if it does when it is orthotropic, the principal axes of the material bear no necessary relationship whatsoever to those of the stress state in general. Stresses in the material's principal axes are usually not the principal stresses as they can be accompanied by shear components.

When generic assumption 2 was adopted in the failure theories for anisotropic composite, it was to support the identification of failure modes such that fibre failure was independent of stresses transverse to the fibres and matrix failure is independent of the direct stress along the fibres. In either case, the fracture plane is not elected by the stress state. The Mohr criterion offers no support whatsoever to the validity of this assumption for such applications. This was probably the reason why when the assumption was made by Hashin [5], direct reference to Mohr's work was avoided, as discussed in [15]. No due diligence has ever been exercised and yet it has never been challenged in the literature. Rather, it has been taken for granted 
ever since. It is therefore a serious cause for concern. In the meantime, contradictions can be found. For instance, although the Puck criterion [6] for UD composites rested heavily on the Hashin criterion of its matrix failure modes [6], it concluded that the transverse direct stresses contributed to fibre failure.

The lack of isotropy has now been shown as a rather rigid wall in front of any serious attempt to stretch generic assumption 2 of the Mohr criterion to modern anisotropic composites. This has been addressed appropriately but separately [16], since it is beyond the scope of the present paper which concentrates on the Mohr criterion itself to eliminate any ambiguity associated with it.

\section{The tensile part of the failure envelope implied by the generic assumptions}

\subsection{Implication of the generic assumptions on triaxial tensile strength}

The Mohr criterion and the failure envelope as shown in Fig. 2 in this appearance is a typical presentation often employed in literature when introducing the Mohr criterion, e.g. [4,9], involving major Mohr's circles for uniaxial tension (UT, to stand for the Mohr's circle as well), pure shear (PS) and uniaxial compression (UC). There has been little further information offered on the tensile side of the envelope, especially in relation to triaxial tension. In Mohr's original paper, it only stated that the failure envelope should be closed on the tensile side and therefore the envelope intersected with the $\sigma$-axis at point corresponding to the major Mohr's circle at failure under hydrostatic tension, which had shrunk to a point at A as marked in Fig. 4. The discussion to follow will hopefully provide some insight to this aspect of the failure envelope based on well-established experimental observations and rational reasoning.

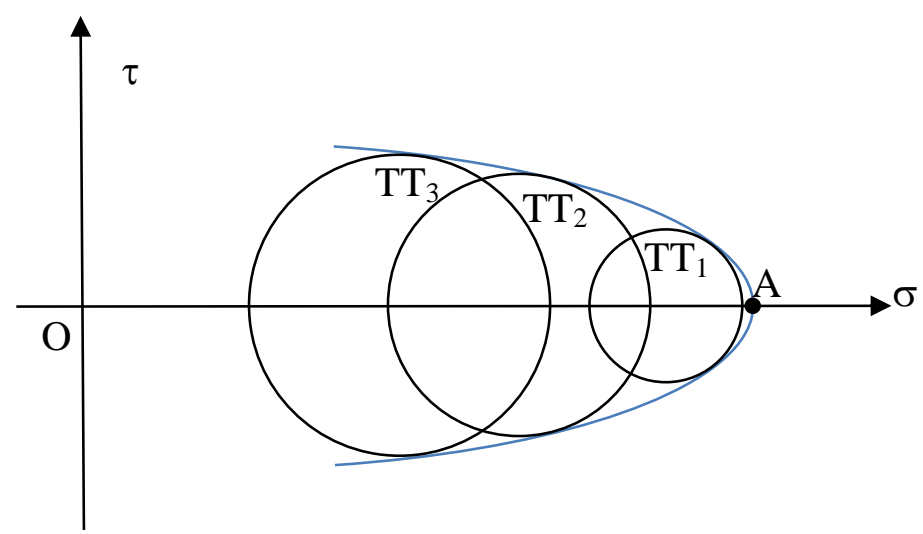

Fig. 4 The tensile end of the envelope for triaxial tensile stress states 
As a fair comment, generic assumption 4 has not attracted sufficient discussion in the literature. Users tend to simplify the envelope into a straight line as Mohr did [3] straightaway before any useful information is revealed. Available discussions in the literature are mostly on the compressive side, given a physically plausible position that materials tend to exhibit infinite strength against hydrostatic pressure. A little elaboration of the envelope in conjunction with inference III unravels a considerable amount of information about it on the tensile side as will be presented below.

It should be pointed out that the existence of the failure envelope is an assumption without which the Mohr criterion would not stand. There would never be sufficient evidence to prove its existence. Otherwise, there would not be any need to make such an assumption. Logically, to disprove its existence, one only needs a single exception contradicting the condition of existence. The Mohr criterion will not be able to afford such an exception. For instance, if the major Mohr's circle at failure under a stress state could be found located completely inside of another for a different stress state, then it would prevent the envelope touching the inner one. This alone would be sufficient to dismiss the existence of such an envelope. Putting it the other round, if there exists an envelope, no major Mohr's circle at failure under any stress state should be contained completely inside another. This will be the main premise supporting the argument to be made.

To facilitate the discussion below, the strength of the material under triaxial tension will be referred to as the value of the $1^{\text {st }}$ principal stress when the material fails. If the envelope exists, the strength under hydrostatic tension, i.e. point $\mathrm{A}$ in Fig. 4, will be no lower than that for any triaxial tensile stress state, e.g. that represented by Mohr's circle TT $_{1}$ in Fig. 4 of a $\sigma_{1}: \sigma_{3}$ ratio greater than 1 , as otherwise the major Mohr's circle at failure under hydrostatic tension would be contained inside $\mathrm{TT}_{1}$.

Similarly, $\mathrm{TT}_{1}$ cannot be contained inside $\mathrm{TT}_{2}$ of a higher $\sigma_{1}: \sigma_{3}$ ratio than that of $\mathrm{TT}_{1}$, and so on. Since none of these stress states can be physically tested reliably, apart from the qualitative statement made above, one cannot quantify anything, e.g. how far point A is away from $\mathrm{TT}_{1}$, and how much $\mathrm{TT}_{1}$ sticks out of $\mathrm{TT}_{2}$, and so on. However, the position will change once a well-established and physically testable case is incorporated into the picture in the next subsection. 
3.2 Observation of uniaxial tensile tests and its implication on the failure envelope

Uniaxial tensile tests are undoubtedly the most well-established type of experiments. For brittle materials, the fracture plane is usually fairly straight and perpendicular to the axis of the specimen and hence to the direction of the $1^{\text {st }}$ principal stress, as shown through the tested samples in Fig. 5 with auxiliary rectangles incorporated to help with the assessment of the orthogonality of the fracture plane to the axis of the specimen. If one approximates it as being perpendicular, i.e. the direction of the $1^{\text {st }}$ principal stress will be normal to fracture plane, according to inference III, the fracture plane should be associated with the point of Mohr's circle on the $\sigma$-axis at $\sigma_{1}$, i.e. point B in Fig. 6. The failure envelope should be tangential to UT at B. Locally, the failure envelope would look like the curve in dashdouble-dot chain (blue) as shown in Fig. 6, i.e. the failure envelope embraces UT and is tangential to UT at point B, rather than hanging over UT as sketched in Fig. 2. Points B should coincide with $\mathrm{A}$ if the failure envelope exists because the intersection between the failure envelope and the $\sigma$-axis must be unique.

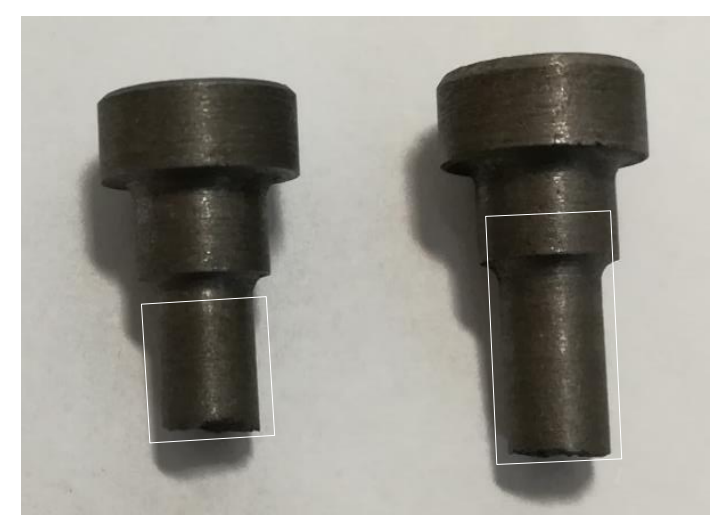

Fig. 5 Tested cast iron specimens under uniaxial tension

Even if one insists that there is a small inclination of the fracture plane in Fig. 5 beyond any experimental uncertainties, it should only be by a very small angle, denoted as $\alpha$. The failure envelope should then touch UT on both sides of the $\sigma$-axis at the circumferential angle of $\alpha$ as indicated. In this case, the failure envelope would look like the dashed curve (red), intersecting the $\sigma$-axis at point A, i.e. the hydrostatic tensile strength, away from point B. Given the fact that from the practically observed fracture planes as shown in Fig. 5, the inclination, if any, must be small enough and hence $\alpha$ is not expected to be anything more 
than a few degrees. Angle $\alpha$ as sketched in Fig. 6 would therefore have been excessively exaggerated in order for angle $\alpha$ to be visible in Fig. 6. With a sufficiently small $\alpha$, the tangential points on the major Mohr's circle on both sides of the $\sigma$-axis are close to each other and, therefore, the tip A of the failure envelope cannot be too far away from B, given that envelopes are supposed to be continuous and smooth in general, being an envelope mathematically. In other words, the distance between A and B must be rather small, if it does not vanish.

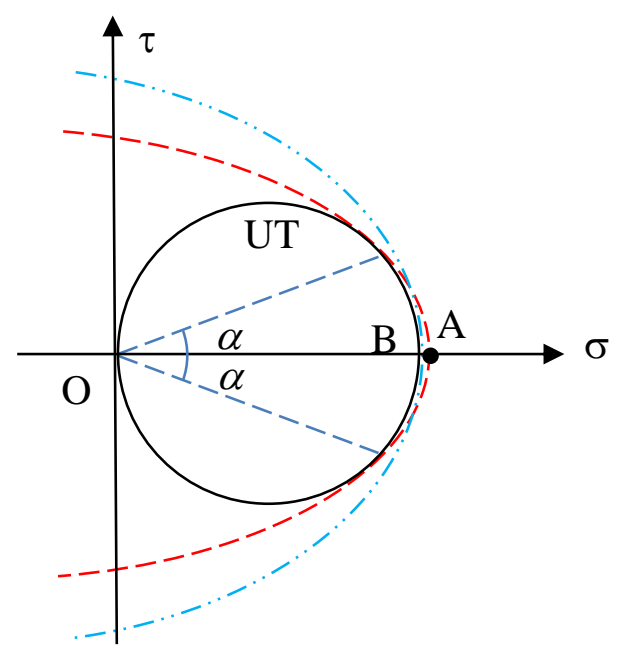

Fig. 6 The tensile end of the envelope considering the uniaxial tensile stress state

Given this argument, one can conclude that all major Mohr's circles for triaxial tension states as shown in Fig. 4 under different stress ratios should have their right hand sides clustered within a marginal distance between $\mathrm{A}$ and $\mathrm{B}$, although their precise magnitudes cannot be quantified. As an approximation, neglecting the distance between $\mathrm{A}$ and $\mathrm{B}$, i.e. the small inclination, if any, of the fracture plane resulting from uniaxial tension, would save a lot of hassles from the unknown triaxial tensile behaviour of materials without causing excessive errors in this respect. If so, the failure envelope on the tensile side should terminate at UT, nothing overhanging as shown in Fig. 2. In other words, materials fail under triaxial tension at the same strength as under uniaxial tension with the fracture plane perpendicular to the first principal stress. The conditions behind this conclusion are generic assumptions 4 , inference III, and established experimental observations of fracture planes under uniaxial tension. This marks the first ever clear statement on the appearance of the tensile part of failure envelope mostly associated with triaxial tensile stress states and this has been achieved rationally. 
As a 'sanity' check, this conclusion agrees with a commonly accepted view that the maximum tensile stress criterion applies well to brittle materials, bearing in mind that all materials tend to fracture in a brittle manner subjected to triaxial tension and the presence of triaxial tension can only make brittle materials even more brittle. It is certainly consistent with all aspects of the Mohr criterion as elaborated above. If the fracture plane is perpendicular to $\sigma_{1}$, then only $\sigma_{1}$ will be exposed on the fracture plane and $\sigma_{2}$ and $\sigma_{3}$ should not make any difference according to assumption 2 .

\subsection{The representativeness of the Mohr's circle for pure shear}

A further inference can be deduced if one considers the pure shear stress state. Use will be made of another well-established experimental observation on the torsion of circular-crosssectioned shafts of brittle materials where the spiral fracture surfaces are consistently observed, as shown in Fig. 7. With the help of the right angle isosceles as included in Fig. 7 to identify the $45^{\circ}$ characteristics, the difference between the fracture surface and the $45^{\circ}$ orientation to the axis of the specimen should not be anything more than a few degrees, if any. Taking a square element out of the surface of the specimen, it is subjected to pure shear. The major Mohr's circle for the pure shear stress state is often considered as one of the few easily accessible test cases as shown in Fig. 2. Again, the discussion here will shake the topology of the failure envelope as sketched in Fig. 2 fundamentally. It is well-known that the fracture of brittle materials under pure shear is primarily due to the tensile stress at $45^{\circ}$ and the fracture surface is at about $45^{\circ}$ to the action plane of the shear stress and hence more or less perpendicular to the $1^{\text {st }}$ principal stress. Taking it to be perpendicular to the $1^{\text {st }}$ principal stress as an approximation, the fracture plane will be found at point $\mathrm{C}$, as indicated in Fig. 8, which should be identical to $\mathrm{A}$ if the intersection between the envelope and the $\sigma$-axis is meant to be unique. Therefore, in Fig. 2, understanding it is an illustrative sketch, one would expect angle $\beta$ which is between the fracture plane and the $1^{\text {st }}$ principal stress to be close to $0^{\circ}$ rather than $45^{\circ} . \beta=45^{\circ}$ means that fracture plane would coincide with the action plane of the shear stress, which contravenes observations as shown in Fig. 7. For the fracture plane to be close to the direction perpendicular to the $1^{\text {st }}$ principal stress, i.e. $\beta$ being very small, the tangential points of the failure envelope to PS should not be far from point $\mathrm{C}$, implying that PS should mostly contain UT, as illustrated in Fig. 8 with necessary exaggerations for visualisation. The argument is the same as in the case for uniaxial tension in the previous subsection. This also suggests that over the range of stress ratios from $\sigma_{1}: \sigma_{3}=1:-1$ to $1: 0$, the 
strengths will be all clustered between point $\mathrm{C}$ (pure shear) and B (uniaxial tensile), bearing in mind that $\mathrm{B}$ is between $\mathrm{A}$ (hydrostatic tension) and $\mathrm{C}$ and that $\mathrm{A}$ and $\mathrm{C}$ are close enough to each other.

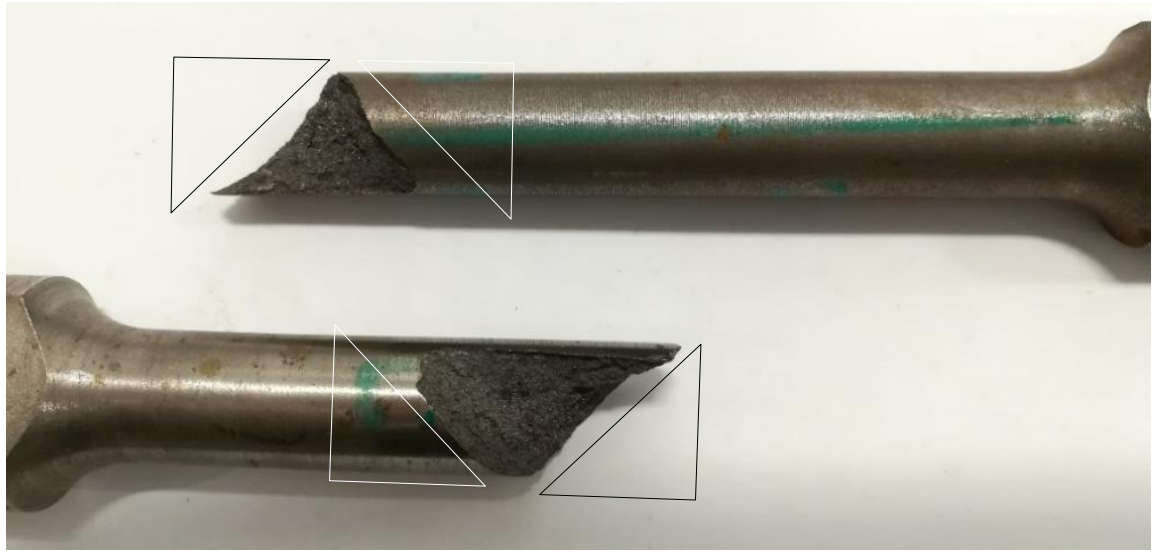

Fig. 7 Tested cast iron specimens under torsion

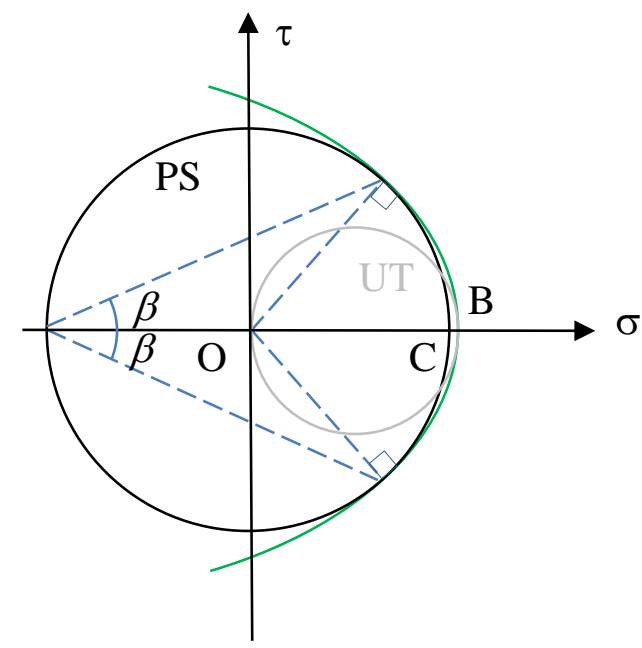

Fig. 8 The relationship between the failure envelope and the Mohr's circles for pure shear and the uniaxial tension

Also as a 'sanity' check, test data from pure shear of brittle materials through torsion tests tend to suggest that its strength is reasonably close to that of the uniaxial tension, i.e. the part of UT sticking out of PS is insignificant, given a wide scatter for these measured strengths due to the brittleness of the material.

Given the argument above, one should expect that PS will play a dominant role in constructing the failure envelope on the tensile side, not the UT. If one neglects the difference between the strengths represented by points A, B and C, the failure envelope will 
terminate at PS on the tensile side, as sketched in Fig. 9. Except for the approximation of the location of point $\mathrm{A}$, the conclusion obtained here is once again based on generic assumption 4 , inference III, and established experimental observations of fracture planes, but this time, under torsion.

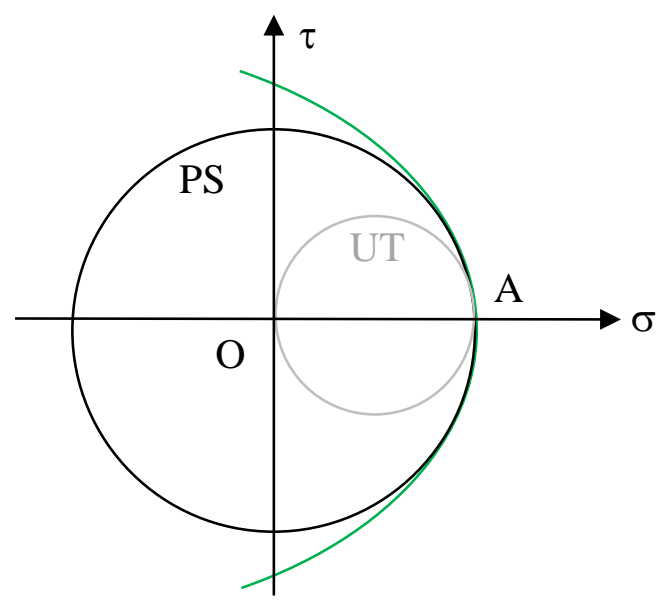

Fig. 9 A simplified way of constructing the failure envelope on the tensile side from the Mohr's circle for pure shear

Since the above obtained conclusion is rationally based on the generic assumptions of the Mohr criterion and well-established experimental observations of fracture surfaces on torsion tests, if one was not happy with it, it would point directly to the associated assumptions, as the experimental observations quoted are unlikely to change, provide that the material is brittle, homogeneous and isotropic.

A similar configuration of the failure envelope on the tensile side was also presented in [11] but it was obtained as one of the special cases when the strength properties of the material happen to take certain values, whilst it has been argued in the present paper that this configuration is generally applicable provided that the generic assumptions hold.

\section{A reasonable construction of the failure envelope}

The arguments made in previous two sections can be summarised as follows. PS should not completely contain UT. On the other hand, if UT sticks out of PS, it will only be by a very marginal part, given the orientations of the fracture planes in these two loading cases, both approximately perpendicular to the $1^{\text {st }}$ principal stress. The similar position can be argued between UT and that of hydrostatic tension. The approximations made on point $\mathrm{A}$ in the 
previous section were two folds, simplification for applications and easiness for delivering the arguments. Without resorting to these approximations, one would need minimum of three strength data, i.e. those from uniaxial tension, pure shear and uniaxial compression tests in order to construct a failure envelope.

Assuming that one has UT, PS and UC only for a material, three conditions are available, i.e. the failure envelope will be tangential to each of them, as depicted in Fig. 10 with necessary exaggerations. These three conditions can be used to determine three parameters. Of course, before any material data have been employed, basic 'sanity' checks should be made to them, because these data are bound to be widely scattered due to the brittleness of the material. Taking any unrepresentative data is an easy recipe for confusion. In general, for brittle materials, the uniaxial compressive strength (measured as the absolute value of the $3^{\text {rd }}$ principal stress) should be substantially higher than the pure shear strength and the uniaxial tensile strength. The uniaxial tensile strength should be no less than the pure shear strength. Ideally, the uniaxial tensile strength should not be excessively greater than the pure shear strength as argued in subsection 3.3. Any anomaly should be carefully assessed in conjunction with the orientation of the observed fracture plane. Appropriate means of data processing should be applied to keep experimental scattering out of the picture.

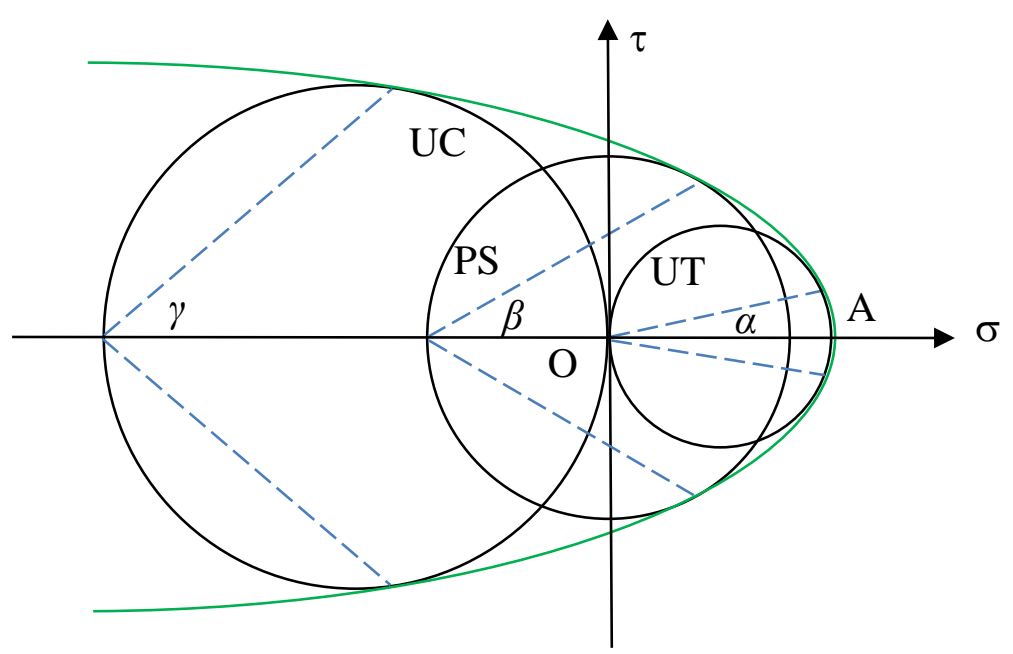

Fig. 10 A rational failure envelope for the Mohr criterion (not to scale, in particular the location of the tangential points)

Given the existing knowledge about the likely shape of the failure envelope, the following equation can be adopted as the form of the failure envelope with three constants $\eta, \lambda$ and $\xi$ to be determined by the three tangential conditions as stated above. 
$\tau^{\eta}=\lambda \sigma-\xi$

where $\sigma$ and $\tau$ are the direct and shear stresses on a plane. Constant $\eta$ must be greater than 1 for the envelope to be curved inwards on the compressive side to avoid any scenario of infinite strength other than hydrostatic compression to avoid the anomaly revealed in [2]. The higher the value of $\eta$, the flatter the envelope on the compressive side. In this respect, a sufficiently high value of $\eta$ is desirable qualitatively. In case of $\eta$ not being an integer, $\tau^{\eta}$ will lose its mathematical definition at $\tau \leq 0$. For the sake of completeness, the part on the negative side of $\tau$ can be produced as a mirror reflection of that on the positive side about the $\sigma$-axis whilst a supplementary definition can be imposed at $\tau=0$, such that $\left.\tau^{\eta}\right|_{\tau=0}=0$ to maintain the continuity of the envelope at its tip. The supplementary point does not seem to upset anything as it happens to correspond to the hydrostatic tension case where no experimental data would be possible anyway. Such defined failure envelope will imply the strength for hydrostatic tension to be $\xi / \lambda$ which should be no less than the uniaxial tensile strength. This should provide a reasonably rational failure envelope for the Mohr criterion for isotropic and brittle materials where there would be no obvious contradiction anymore if the generic assumptions 1-5 as stated in Section 2 hold. Any inconsistency readers might detect in future would therefore directly point to one flaw or another in one or more basic or generic assumptions of the Mohr criterion. Apparently, (1) is of an empirical nature, which can accommodate 3 coefficients to be determined without any anomaly but it is by no means a unique expression one can adopt.

The form of (1) resembles that of the $\mathrm{JH}-2$ model [17] widely employed in impact engineering although $\mathrm{JH}-2$ was given as the relationship between the direct stress and the hydrostatic pressure as the hardening rule of plasticity instead of shear stress and the direct stress on the fracture plane. The key features of these relationships based on power law are

i) The curves exhibit infinite slope at intersection with the horizontal coordinate axis;

ii) They do not have asymptotes and therefore will never level off toward the extreme on the compressive side of the curve.

The first feature was of little significance as far as the applications of JH-2 model were concerned since they focused primarily on materials' behaviour in presence of compressive hydrostatic stress. There has been so little known on the tensile hydrostatic stress side until 
the emergence of the insight achieved in this paper. It is now known how important this feature is as one of the major characteristics of the failure envelope for the Mohr criterion on the tensile side.

The second feature could be critical, as evidences seemed to suggest a levelling off trend at high hydrostatic stresses [12]. To reflect this trend, one would find an exponential relationship, such as those employed in [12] as an extended form of C-M and also in [18] as the hardening rule of plasticity.

Given the understanding achieved in this paper and experimental data as shown in $[12,13]$, in order to obtain full field applicability from hydrostatic compression to hydrostatic tension, it would be good practice to have a function defined in a piece-wise manner, i.e. a power function on the tensile side and an exponential function on the compressive side. One can certainly find a tangential point between the two curves to ensure a continuous and smooth transition from one to another. This will be further elaborated later in a slightly simpler scenario.

If one is prepared to take some approximations by assuming that the strengths for PS and hydrostatic tension collapse to the same value as that for UT, one only needs two input data, the strength for UC and PS. As PS is usually more demanding to obtain than UT, one may use UT to construct PS by taking the diameter of UT as the radius of PS. Given that the intersection with the $\sigma$-axis has been determined by PS, there is only one condition available, i.e. the failure envelope must be tangential to UC. In this case, a simplified expression for the failure envelope can be given as

$\tau^{n}=\lambda(\sigma-T)$

where $T$ is the tensile strength of the material. For relationship (2) to be unique, $n$ should be chosen as the lowest even number which spares (2) cutting across PS. The only constant $\lambda$ to be determined should be obtained using the tangential condition to UC. Ideally, the tangential points should be close to the top and bottom of UC because established experimental observations of the fracture planes of brittle materials under uniaxial compression as shown in Fig. 11 tend to demonstrate a clear inclination at about $45^{\circ}$ (with reference to the right angle isosceles included) to both $\sigma_{1}$ and $\sigma_{3}\left(\sigma_{1}\right.$ happens to be zero in this case). In other word, the failure envelope around the tangential point should be reasonably flat (i.e. $\gamma \approx 45^{\circ}$ ) with negative slope of small magnitude for the top half lobe of 
the failure envelope. This is perhaps as simple as one could go to bring forward a failure envelope for the Mohr criterion with minimum requirement for test data, whilst avoiding any pronounced inconsistency.

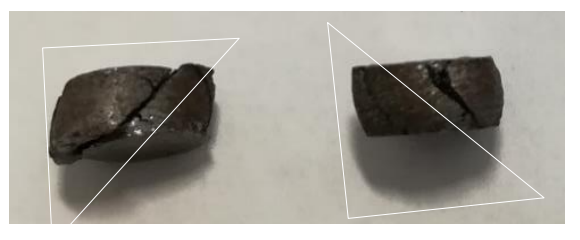

Fig. 11 Tested cast iron specimens under uniaxial compression

Along the same line of piece-wise defined curve for the complete field, employing the exponential curve of the EMC model [12] for the compressive side and PS on the tensile side, an appropriate approximation could be as

$$
\tau=\left\{\begin{array}{cc}
\sqrt{T^{2}-\sigma^{2}} & \text { if } T \cos \varphi \leq \sigma \leq T \\
A+B e^{-K \sigma} & \sigma<T \cos \varphi
\end{array}\right.
$$

where $A, B$ and $K$ are the constants to be determined by curve fitting to the available test data on the compressive side and $\varphi$ is the central angle of the PS defining the tangential point. Apparently, to make sure the continuity and smoothness between these two segments, the exponential function should be fitted to the test data with this consideration incorporated. Detailed exploration along the line is left for readers to fulfil as it is obviously beyond the scope of the present paper.

\section{Linearized failure envelope in the Mohr criterion to assist a critical appraisal of the Coulomb-Mohr criterion}

Given the simplification as outlined at the end of the previous section, the failure envelope in the Mohr criterion should never be linearized, if one wishes to capture the key features of the failure envelope and hence to maintain the representativeness of the theory as rationalised above in this paper. However, it will be done below regardless, not to make much sense out of the Mohr criterion itself, but to assist an appropriate appraisal of C-M.

Based on the discussions made in previous sections, the linearized failure envelope as a pair of straight lines should be constructed to be tangential to UC and PS. This does not prevent 
one constructing PS from UT as described earlier as depicted in Fig. 12. This will be a far more logical solution than using UT directly as has been the case for the C-M hitherto.

The straight lines as the failure envelope should not be extended beyond the tangential point to the PS on the tensile side, whilst on the tensile side an appropriate approximation of the failure envelope should be given simply by that part of the PS. In this sense, the failure envelope is only partially linearized, since the full linearization is deemed to be inappropriate as argued in previous sections. The complete failure envelope should be given as the dashed profile (red) as shown in Fig. 12. Because of the use of straight lines for the envelope on the compression side, the anomaly as cited in [2] remains present in this linearized version. The nonlinear part on the tensile side reproduced the maximum principal stress criterion for any stress state of a stress ratio $\sigma_{1}: \sigma_{3}=1: k(1>k>-1)$.

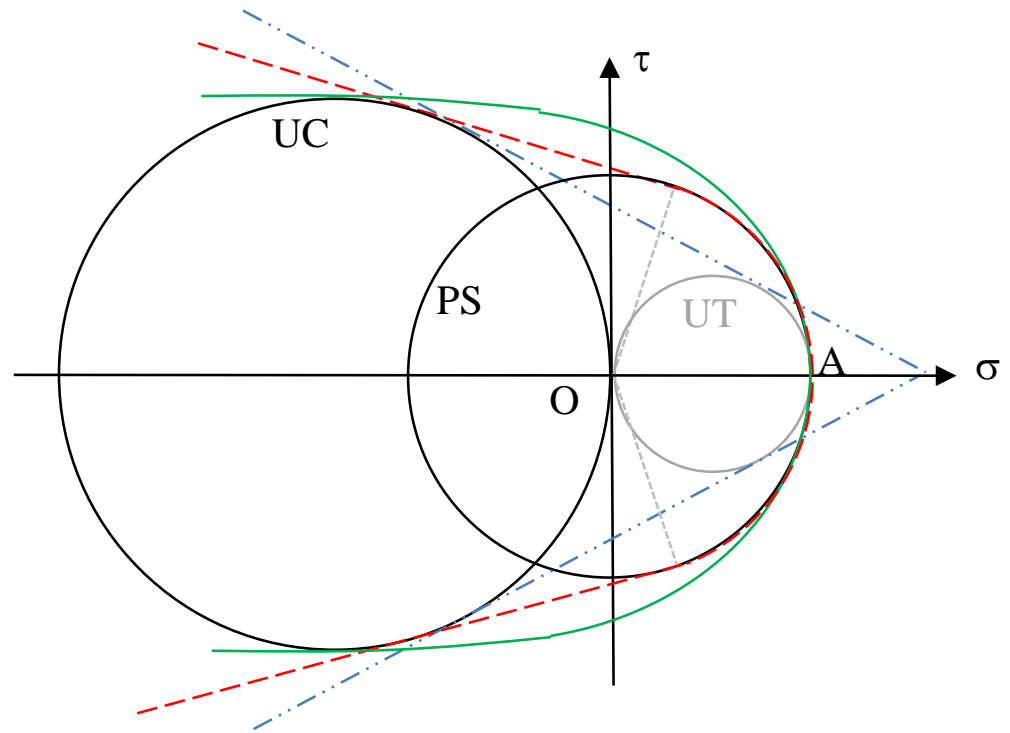

Fig. 12 Failure envelopes for the simplified Mohr (solid), the linearized Mohr (dashed) and the C-M (dash-double-dot chain)

The orientation of fracture planes should never be obtained from the linearized failure envelope as opposed to inference III. Given the elaboration in Sections 2 and 3, the more realistic failure envelope should look that as shown in Fig. 12 as the envelope in solid line (green) to which inference III applies. Manipulation to a function (the envelope) tends to result in excessive distortion to its derivative. Realistic orientation of fracture plane represented by its outward normal should be about $45^{\circ}$ to the $1^{\text {st }}$ principal stress for stress states ranging from hydrostatic compression up to uniaxial compression, i.e. $\sigma_{1}: \sigma_{3}=-\infty:-\infty$ to 0:-1. Between UC and PS, i.e. over the range of stress ratios from $\sigma_{1}: \sigma_{3}=0:-1$ to $1:-1$, the 
angle varies from about $45^{\circ}$ gradually to about $0^{\circ}$. From PS up to hydrostatic tension, i.e. from $\sigma_{1}: \sigma_{3}=1:-1$ to $1: 1$, it should stay at about $0^{\circ}$. In other words, all changes in the orientation of fracture plane take place between UC and PS from about $45^{\circ}$ to about $0^{\circ}$ whilst remaining more or less constant on both sides. Such variation can never be properly reflected by the linearized failure envelope. Mathematically, the best one can conclude is that over the full range of stress ratios, the linearized failure envelope has at least one chance of hitting the correct orientation of the fracture plane at a certain stress ratio according to Roll's meanvalue theorem [19], which is far from being informative.

The difference between the 'linearized' Mohr criterion as presented above and the C-M can be clearly demonstrated in Fig. 13 where the failure envelope in the plane of the two principal stresses under a plane stress condition. The parts of the envelope in the $1^{\text {st }}$ and the $3^{\text {rd }}$ quadrants are primarily the input data. It is obvious that, whilst the maximum stress criterion represents an overestimate of the strength, C-M underestimates the strength in the $2^{\text {nd }}$ and $4^{\text {th }}$ quadrants.

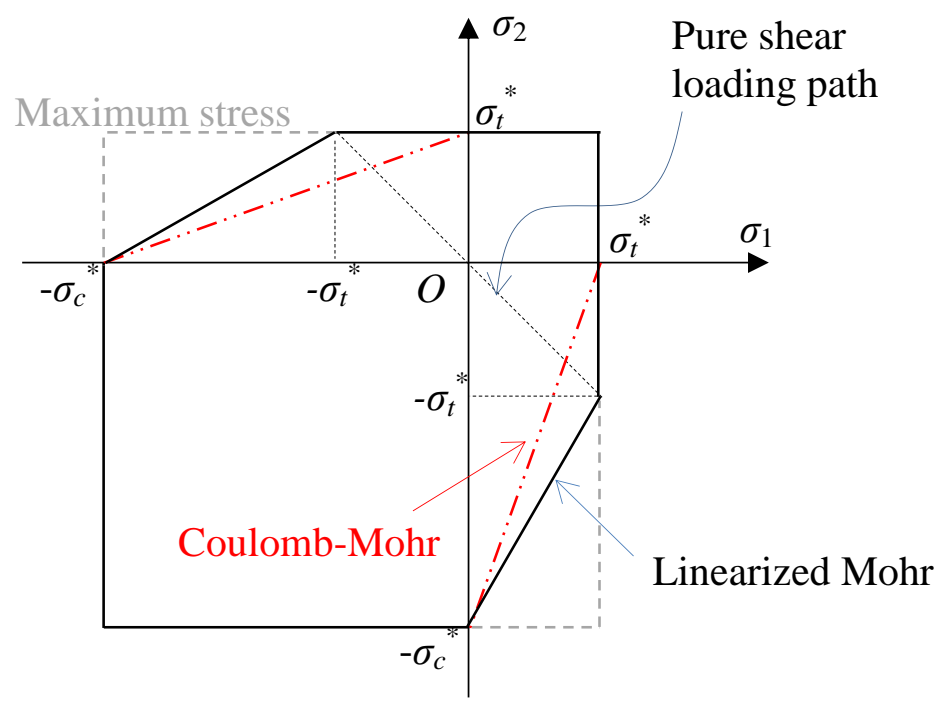

Fig. 13 Comparison of the Mohr criterion with linearized failure envelope, the CoulombMohr criterion and the maximum stress criterion for a plane stress state

The use of UT in place of PS to construct the linearized failure envelopes represents a fundamental oversight of well-established experimental observations and gross negligence of realistic relationship between uniaxial tension and pure shear. Further extension of it to the triaxial tensile side contradicts the generic assumptions of the Mohr criterion and established experimental observations. Correcting these mistakes should never be an achievement one 
could be proud of, given that they were made over a hundred years ago. However, it will not be acceptable if they are left uncorrected as they would keep misleading users in future otherwise.

An appropriate appraisal of C-M can now be made rationally as follows. On the compressive side, given the anomaly as cited in [2], it cannot be trusted for triaxial compression. It severely underestimates the strengths for stress states around pure shear in the middle ground. It was probably not meant to be used at all on the tensile side as it was nothing more than a blind extrapolation. Applicability of $\mathrm{C}-\mathrm{M}$ is limited to those close to two stress states, uniaxial tension and compression, where strengths can be reasonably predicted. This is little more than reproduction of input data. Even for them, the fracture planes are mispresented. In fact, the fracture plane prediction is wrong for almost all stress states. The failure envelope employed in C-M is apparently a gross misrepresentation of well-established experimental data and available observations, needless to mention that $\mathrm{C}-\mathrm{M}$ allows a single fracture angle to the direction the $1^{\text {st }}$ principal stress for all stress states, which is wrong at least for uniaxial tension, let alone others. With hindsight, as a material failure criterion, it probably should not have been introduced to engineering and certainly should be phased out from textbooks in future. If a simple version of the Mohr criterion has to be presented as an introduction, e.g. in a textbook, it should be the partially linearized Mohr criterion as proposed above with a clear warning about the anomaly under triaxial compression as revealed in [2] whilst ignoring the predicted fracture plane.

\section{Conclusions}

The assumption systems for the Mohr criterion have been streamlined. The relationship between the orientation of the fracture plane and the failure envelope has been logically deducted, whilst it has been avoided or taken for granted without any justification in the literature. The particular generic assumption that 'failure is determined by the stresses exposed on the fracture surface' has been scrutinised and it has been revealed that its applicability is strictly restricted to isotropic materials. Therefore, its adoption in the establishment of popular failure criteria for modern anisotropic fibre reinforced composites was unfounded and out of context. Although the assumption helped the establishment of the Mohr criterion and proved to be useful, it probably had gone as far as it could.

As logical implications of the generic assumptions made in the Mohr criterion, the tensile part of the failure envelope has been narrowed down to a small neighbourhood around the 
major Mohr's circle for pure shear at failure and can therefore be satisfactorily approximated by it for stress state over a wide range from pure shear up to hydrostatic tension, i.e. over stress ratios from $\sigma_{1}: \sigma_{3}=1:-1$ to $1: 1$. This has been obtained based on rational deduction of the generic assumptions assisted by well-established experimental observations on uniaxial tension and pure shear.

It has also established that the Mohr's circle for pure shear stress state is far more representative than its uniaxial tensile counterpart in the construction of the failure envelope. The Coulomb-Mohr criterion has been critically and appropriately appraised and the outcome is that it is mostly unreliable and should be phased out completely.

\section{Acknowledgement}

The author wishes to express his gratitude to the comments made by Prof Puhui Chen of NUAA, China on an early draft of this paper.

\section{References}

[1] Coulomb, C. A. (1773) Essai sur une application des régles de Maximis \& Minimis à quelquels Problèmes de Statique, relatifs à l'Architecture. In Mémoires de Mathématique et de physique préfenté à l'Acádemie Royale des Sciences, par divers Savans, \& lûs dans fes Affemblées, 7:343-382

[2] Christensen, R.M. (2013) The Theory of Materials Failure, Oxford University Press, Oxford

[3] Mohr, O. (1900) Welche Umstände bedingen die Elastizitätsgrenze und den Bruch eines Materials? Civilingenieur, 44(45):1524-1530 \& 44(46):1572-1577

[4] Timoshenko, S. (1963) Strength of Materials, Part II: Advanced Theroy and Problems, $3^{\text {rd }}$ edn, D. Van Nostrand Company, Inc., New York

[5] Hashin, Z. (1980) Failure Criteria for Unidirectional Fiber Composites, Journal of Applied Mechanics, 47:329-334

[6] Knops, M. (2008) Analysis of Failure in Fiber Polymer Laminates — The Theory of Alfred Puck, Springer, Berlin

[7] Pinho, S.T., Dávila, C.G., Iannucci, L. and Robinson, P. (2005) Failure models and criteria for FRP under in-plane or three-dimensional stress states including shear non-linearity, NASA/TM-2005-213530, NASA Langley Research Center, Hampton, VA 23681, USA 
[8] Paul, B. (1961) A modification of the Coulomb-Mohr theory of fracture, J. Appl. Mech., 28:259-268

[9] Timoshenko, S.P. (1983) History of Strength of Materials, Dover Publications, New York

[10] Yu, M.H. (2004) Unified Strength Theory and Its Applications, Springer, Berlin

[11] Gu, J. and Chen, P. (2018) A failure criterion for isotropic materials based on Mohr's failure plane theory, Mechanics Research Communications, 87:1-6, doi: 10.1016/j.mechrescom.2017.11.008

[12] Shafiq M. and Subhash G. (2016) An extended Mohr-Coulomb model for fracture strength of intact brittle materials under ultrahigh pressures, J. American Ceramic Society, 99(2):627-630, doi: 10.1111/jace.14026

[13] Bavdekar, S. and Subhash, G. (2018) Comparison of pressure-sensitive strength models for ceramics under ultrahigh confinement, Int. J. Impact Eng., 118:60-66, DOI: 10.1016/j.ijimpeng.2018.04.007

[14] Bavdekar, S., Subhash, G. and Satapathy, S. (2019) A unified model for dwell and penetration during long rod impact on thick ceramic targets, Int. J. Impact Eng, 131:304-316, DOI: 10.1016/j.ijimpeng.2019.05.014

[15] Li, S. and Sitnikova, E. (2018) A critical review on the rationality of popular failure criteria for composites, Composites Communications, 8:7-13, doi: 10.1016/j.coco.2018.02.002

[16] Li, S. and Sitnikova, E. (2020) A critical appraisal of the key assumption in the Hashin failure criterion, submitted to Composites Science and Technology for possible publication

[17] Johnson G. R. and Holmquist T. J. (1994) An improved computational constitutive model for brittle materials, AIP Conference Proceedings, 309(1):981-984, doi: 10.1063/1.46199

[18] Johnson G. R., Holmquist, T. J. and Beissel S. R. (2003) Response of aluminum nitride (including a phase change) to large strains, high strain rates, and high pressures, J. Appl. Phys., 94(3):1639-1646, doi: 10.1063/1.1589177

[19] Korn, G.A. and Korn, T.M. (1968) Mathematical Handbook for Scientists and Engineers, McGraw-Hill, New York 